\title{
REGULAR COSINE FAMILIES OF LINEAR SET-VALUED FUNCTIONS
}

\author{
MASOUMEH AGHAJANI
}

Abstract. This paper is concerned with the properties of regular cosine families of continuous linear set-valued functions defined on convex cones of normed spaces. We consider conditions under which a regular cosine family of continuous linear set-valued functions is continuous and then generalize some recent results on commutativity and Hukuhara's derivative of regular cosine families of continuous linear set-valued functions.

Mathematics subject classification (2010): 54C60, 28B20, 47D09.

Keywords and phrases: Riemann integral of set-valued functions, cosine families, Hukuhara's derivative, linear set-valued functions.

\section{REFERENCES}

[1] M. Aghajani And K. Nourouzi, On Hukuhara's differentiable iteration semigroups of linear setvalued functions, Aequationes Math., 90, 6 (2016), 1129-1145.

[2] M. Aghajani And K. Nourouzi, On the regular cosine family of linear correspondences, Aequationes Math., 83, 3 (2012), 215-221.

[3] C. D. Aliprantis And K. C. Border, Infinite Dimentional Analysis, A hitchhiker's guide. Third edition. Springer, Berlin, 2006.

[4] Z. FEChner AND L. SzÉKelyhidi, Sine and cosine equations on hypergroups, Banach J. Math. Anal., 11, 4 (2017), 808-824.

[5] M. Hukuhara, Intégration des applications mesurables dont la valeur est un compact convexe, Funkcial. Ekvac., 10, (1967), 205-223.

[6] S. Łojasiewicz, An introduction to the theory of real functions, John Wiley and Sons, Chichester, 1988.

[7] E. Mainka-Niemczy K, Multivalued second order differential problem, Ann. Univ. Paedagog. Crac. Stud. Math., 11, (2012), 53-67.

[8] J. R. MunKRes, Topology: a first course, Prentice-Hall, 1975.

[9] K. Nikodem, $K$-convex and $K$-concave set-valued functions, J. Zeszyty Nauk. Politech. Łódz. Mat. 559, J. Rozprawy Nauk., 114, (1989).

[10] M. PIsZCZEK, Integral representations of convex and concave set-valued functions, Demonstratio Math., 35, 4 (2002), 727-742.

[11] M. PiszczeK, On cosine families of Jensen set-valued functions, Aequationes Math., 75, 1-2 (2008), $103-118$.

[12] M. Piszczek, On multivalued cosine families, J. Appl. Anal., 13, 1 (2007), 57-76.

[13] M. PiszcZEK, Second Hukuhara derivative and cosine family of linear set-valued functions, Ann. Acad. Pedagog. Crac. Stud. Math., 5, (2006), 87-98.

[14] H. RȦDSTRÖM, An embedding theorem for space of convex sets, Proc. Amer. Math. Soc., 3, (1952), $165-169$.

[15] A. SMAJDOR, Hukuhara's derivative and concave iteration semigroups of linear set-valued functions, J. Appl. Anal., 8, 2 (2002), 297-305.

[16] A. SMAjdor, Hukuhara's differentiable iteration semigroups of linear set-valued functions, Ann. Polon. Math., 83, 1 (2004), 1-10.

[17] A. Smajdor, On regular multivalued cosine families, European Conference on Iteration Theory (Muszyna-Zlockie, 1998). Ann. Math. Sil., 13, (1999), 271-280. 
[18] A. Smajdor And W. Smajdor, Commutativity of set-valued cosine families, Cent. Eur. J. Math., 12, 12 (2014), 1871-1881.

[19] W. SMAJDOR, Superadditive set-valued functions and Banach-Steinhause theorem, Rad. Mat., 3, 2 (1987), 203-214. 